\title{
Small Aircraft Cockpit Electromagnetic Interference Due to UMTS Signal Propagation
}

\author{
Clifford De Raffaele*, Carl James Debono*, and Adrian Muscat \\ Department of Communications and Computer Engineering \\ University of Malta, Msida, MSD 2080, Malta \\ E-mail: cderaffaele@ieee.org, c.debono@ieee.org, adrian.muscat@um.edu.mt
}

\section{Introduction}

The last decade has seen a drastic revolution in onboard communication and entertainment services, with improvement being registered in terms of convenience, style, content, and also a limited degree of availability on demand. Nevertheless, the increasing proliferation of personal mobile electronic devices, has urged the aviation industry into requiring personalized in-flight services. This is evermore critical, since nowadays aircraft seem to be the last remaining frontier where wireless mobile communications and internet access are not available to the same standard as that of their terrestrial counterparts [1]. Feasibility constraints dictate that the approach most likely to be considered for the provision of wireless connectivity operates by establishing an onboard local area network (LAN) from which a specific base transceiver station (Node B) will serve as a gateway to the terrestrial backbone infrastructure via a satellite link [2].

A number of flight safety considerations must, therefore, precede the implementations of such communication systems. Modern aircraft employ a complex suite of electronic systems for navigation, control and internal communications during operation, all of which must be kept immune from penetration of high-intensity radiated fields (HIRF) as to prevent the occurrence of critical flight incidents due to electromagnetic interference (EMI) [3]. To this end, prior to service engagement, aircraft must undergo a number of certification standards [4], which demand the implementation of costly and time consuming measurement campaigns [5]. The execution of such a technique is however problematic in the private business jet industry, since aircraft models alter significantly between manufacturers and client specifications. Accurate predictions of the propagation characteristics inside an aircraft cabin, however, differ significantly from those emulated by conventional indoor propagation models, demanding the need for dedicated models. Phenomena of multipath propagation are a dominant effect in aircraft due to the dense cluttering of cabin furniture and almost complete shadowing of line-of-sight signals. Furthermore, the particular construction of the fuselage exhibits a waveguide effect for electromagnetism due to the tunnel-like structure involved [6].

This paper models the EMI generated inside the cabin of a small medium-haul aircraft from a Node B antenna providing UMTS service. This is achieved by means of a three-dimensional raytracing algorithm which, with difference from previous cabin implementations [5] [7], considers the volumetric properties of the cabin furniture and their complex electromagnetic material properties, to enhance modeling accuracy via thin film optics physics. Moreover, two distinct simulations are performed with different setup configurations for the antenna locations in a study to reduce implementation costs whilst still providing sufficient coverage to all users onboard. In each scenario, the EMI field incident on the cockpit avionics panel from the Node B is analyzed. 


\section{Propagation Model}

Calculation of the EMI field incident on the inner fuselage structure and in particular on the avionics bay, is performed by the implementation of an area oriented design based on ray launching techniques. This algorithm is able to achieve a computational complexity which is almost independent on the amount of observation points and aircraft size, whilst being linearly dependent on the number of subsequent environment interactions [8]. At $2.1 \mathrm{GHz}$, the signals wavelength is much smaller than the architectural dimensions of passenger aircraft models, thus invoking the implementation of the Geometric Optics hypothesis for the calculation of EM field.

The model takes into consideration a fixed transmission station that operates at a power of $45 \mathrm{dBm}$. Rays from this source are launched randomly via a Monte-Carlo method in spherical angular coordinates to ensure that total coverage of the antenna is emulated in an asymptotical manner. As per definition, the basis of ray tracing involves the calculation of the electromagnetic field at any point to be performed by taking into consideration every ray incident on the location. Electromagnetic field of each ray is derived by accounting for the path loss experienced by the wavefront in the propagation medium with a resolution of eight samples per wavelength. Once the latter impinges on a boundary composed of a discontinuity in media, Fresnel's equations are employed together with the complex properties of each material so as to compute the ratio of reflected power and that transmitted through the new medium. Since materials are composed of irregular surfaces, scattering effects to account for the specular direction of propagation are also implemented to enhance the model's accuracy [9]. A total of 200000 rays are simulated in the scenario through the several propagation mechanisms until each ray reaches a signal strength of $-150 \mathrm{dBm}$, where it is then negligible for the vectorial computation of the total EMI.

\section{Simulation and Results}

The algorithm developed in this paper was used to model a base station onboard a small jet replicating the geometry of the Evektor EV-55 Outback passenger aircraft [10] with a resolution of $2 \mathrm{~cm}$ in all directions on the Maltab ${ }^{\circledR}$ platform. As illustrated in Figure 1, an eight passenger cabin configuration, mainly composed of an aluminum fuselage with acrylic windowpanes furnished by wooden flooring and rows of leather seats, was simulated. Each material was subsequently assigned the respective electromagnetic parameters in order to accurately compute the complex coefficients for the multipath rays that interacted with the cabin environment.

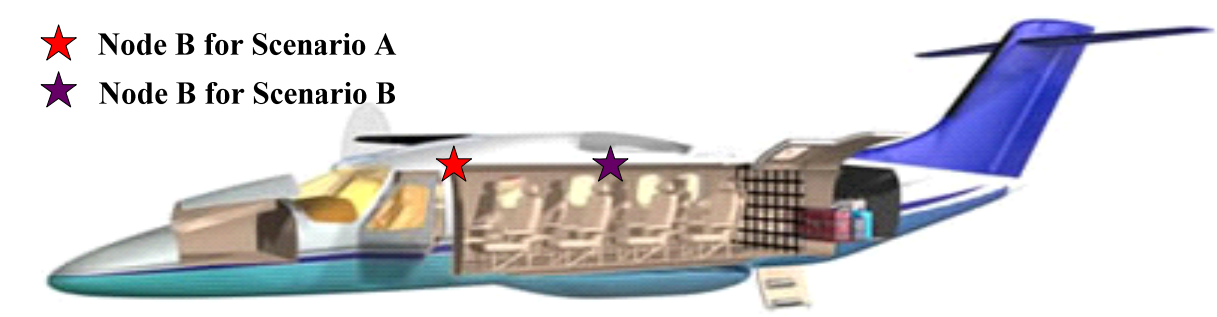

Figure 1: Evektor EV-55 business jet model [10], as included in the electromagnetic propagation simulation.

Two distinct simulations were performed with network coverage provided by means of a single Node B antenna placed in different locations. Scenario A, considers the antenna located just outside the cockpit compartment. This location is favorable due to the proximity of data and cable connections that need to be provided to access node, which would only impose a minimal 
increase in hardware and consequently less additional weight. Figure $2 \mathrm{a}$ demonstrates that this antenna location is able to provide sufficient coverage to the farthest passenger seat inside the cabin. However, the close proximity to the cockpit area also implies that multipath effects from nearby obstacles combine to yield a significant EMI field on the avionics bay, which might pose severe interference issues as evidenced in Figure 2b. Results show that a mean EMI power of $-11 \mathrm{dBm}$ is incident on the instrumentation with certain locations experiencing peaks of $10.8 \mathrm{dBm}$.

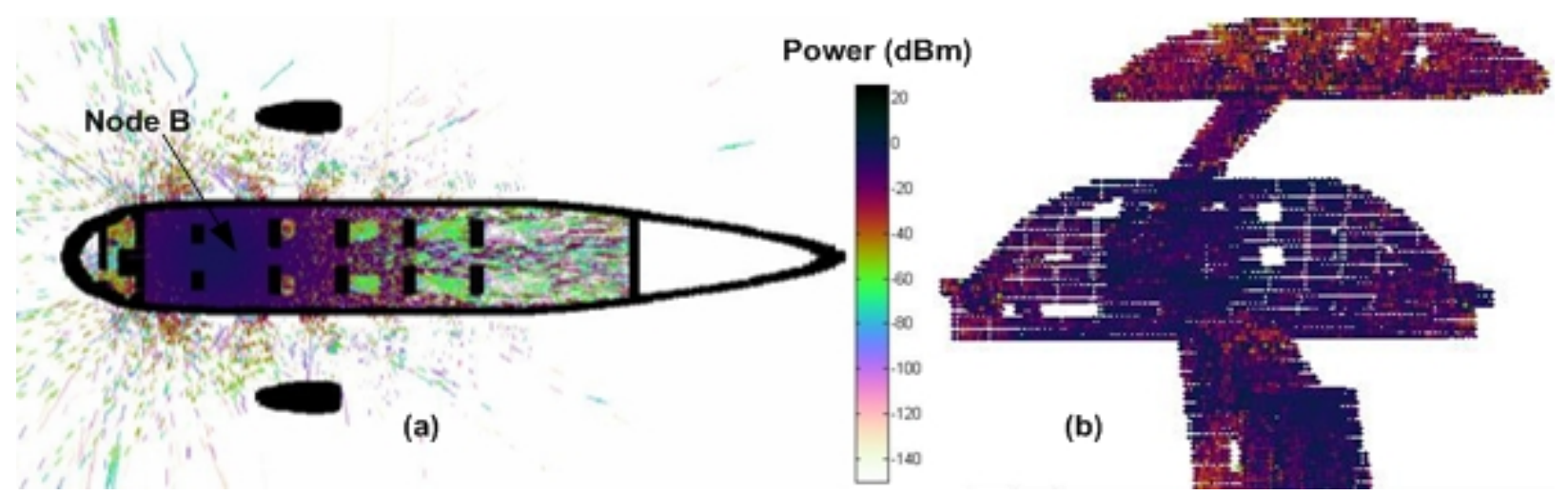

Figure 2: a) Resultant EV-55 propagation map and b) Electromagnetic interference generated on avionics panel from a mid-cabin located Node $B$ antenna.

Simulation B, with propagation coverage depicted in Figure 3a, considers the antenna being placed on the ceiling in the middle of the passenger cabin. It is immediately evident that global coverage of the cabin is improved in this manner, since more line-of-sight paths are present between the Node B antenna and mobile user locations. Figure 3b, also testifies that the EMI field incident on the instrumentation is drastically reduced in this scenario with a mean average power of $-102.5 \mathrm{dBm}$ and peak of $-5.6 \mathrm{dBm}$. This, effect is due to the augmentation in distance as well as the increase in obstructing cabin furniture between the antenna and the avionics panel.

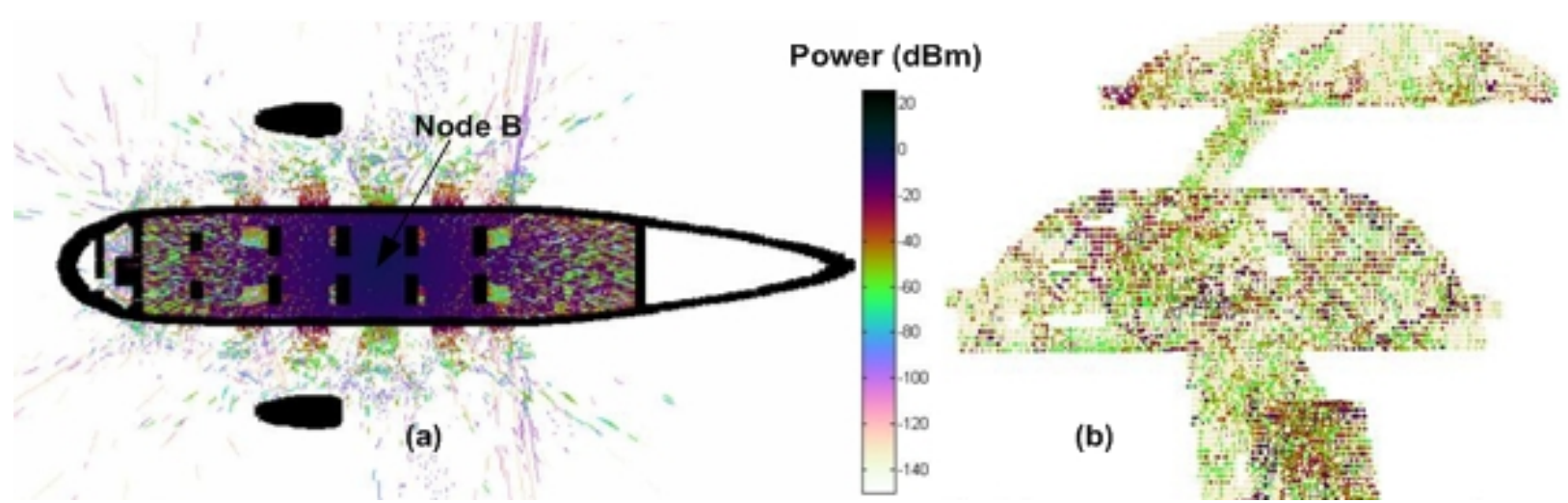

Figure 3: a) Resultant EV-55 propagation map and b) Electromagnetic interference generated on avionics panel from a near-cockpit located Node $B$ antenna.

Upon comparing the different scenarios, it is evident that the implementation of the antenna away from the cockpit area, although implying a compromise on the installation hardware and associated increase in weight, presents a much lower EMI field and should be considered as the better solution. Nevertheless, the accurate simulation modeling performed highlights the compelling need for the development of better shielding. This is even more critical for the cables and systems employed for communications and control. 


\section{Conclusion}

This paper implemented a ray-tracing based algorithm to calculate the EMI generated inside an aircraft cabin with particular emphasis on flight critical equipment such as the cockpit avionics panel. The interference level caused by the deployment of a UMTS Node B antenna for the provision of passenger communication was computed. The model was employed to study the effects of the multipath electromagnetic propagation attained from positioning a Node B antenna in different locations inside a relatively small tunnel shaped fuselage with dense furnishings. The proposed framework is also able to provide an all-encompassing understanding of the generated EMI, whilst offering support for the development of conscientious network design in aircraft.

\section{Acknowledgement}

The authors would like to thank Evektor Aviation for providing a model of the aircraft. This work forms part of the project HIRF SE which is financially supported under the European Community 7th Framework Programme (FP7). The authors are solely responsible for the contents of the paper which does not represent the opinion of the European Commission.

\section{References:}

[1] A. Jahn, et. al. "Evolution of aeronautical communications for personal and multimedia services," in IEEE Communications Magazine, Vol. 41, pp. 36-43, Jul. 2003.

[2] K.D. Lin and J.F. Chang, "Communications and entertainment onboard a high-speed public transport system", in IEEE Wireless Communications, Vol. 9, pp. 84-89, Feb. 2002.

[3] S.V. Georgakopoulos, C.R Birtcher, C.A. Balanis and R.A. Renaut, "HIRF penetration and PED coupling analysis for scaled fuselage models using a hybrid subgrid FDTD(2,2)/FDTD(2,4) method," in IEEE Trans. on Electromagnetic Compatibility, Vol. 45, No. 2, pp. 293-305, May 2003.

[4] RTCA SC-202, "Aircraft design and certification for portable electronic devices (PED) tolerance," RTCA, Inc., Washington, DC, DO-307, Oct. 2007.

[5] K. Chetcuti, C.J. Debono, R.A. Farrugia and S. Bruillot, "Wireless propagation modelling inside a buisness jet", in Proc. of Eurocon 2009, pp. 1640-1645, May 2009.

[6] N.R. Diaz and J.E. Esquitino, "Wideband channel characterization for wireless communications inside a short haul aircraft," in Proc. of the 59th IEEE Vehicular Technology Conf., pp. 223-228, Germany, May 2004.

[7] B.E. Geschwendtner, G. Wolfle, B. Burk and F.M. Landstrofer, "Ray tracing vs. ray launching in 3-D microcell modeling" in Proc. of the European Personal and Mobile Communications Conf., pp 74-79, 1995.

[8] J. Lacik, Z. Lukes and Z. Raida, "On using ray-launching method for modeling rotational spectrometer," in Radioengineering, Vol.17, No.2, pp.98-107, June 2008.

[9] C. De Raffale, C.J. Debono and A. Muscat, "Modeling electromagnetic interference generated by a WLAN system onboard commercial aircraft", in Proc. MELECON 2010, Malta, April 2010.

[10] Evektor Aviation EV-55 website. Available [Online] http://www.evektor.cz/outback. 